\title{
Early assessment of lung aeration using an ultrasound score as a biomarker of developing bronchopulmonary dysplasia: a prospective observational study
}

\author{
Ignacio Oulego-Erroz $\mathbb{D}^{1,2,3} \cdot$ Paula Alonso-Quintela ${ }^{3,4} \cdot$ Sandra Terroba-Seara ${ }^{3,4} \cdot$ Aquilina Jiménez-González $^{4}$. \\ Silvia Rodríguez-Blanco ${ }^{4}$
}

Received: 10 March 2020 / Revised: 27 May 2020 / Accepted: 7 July 2020 / Published online: 14 July 2020

(c) The Author(s), under exclusive licence to Springer Nature America, Inc. 2020

\begin{abstract}
The objective of this study was to assess the predictive value of a lung ultrasound (LUS) score in the development of moderate-severe bronchopulmonary dysplasia (sBPD). This was a prospective observational diagnostic accuracy study in a third-level neonatal intensive care unit. Preterm infants with a gestational age below 32 weeks were included. A LUS score (range $0-24$ points) was calculated by assessing aeration semiquantitatively ( $0-3$ points) in eight lung zones on the 7 th day of life (DOL) and repeated on the 28th DOL. ROC curves and logistic regression were used for analysis. Forty-two preterm infants were included. The LUS on the 7th DOL had an area under the receiver operating characteristic curve (AUROC) of 0.94 (95\% CI: 0.87-1) for the prediction of sBPD (optimal cutoff of $\geq 8$ points: sensitivity 93\%, specificity 91\%). The LUS score was independently associated with SBPD [OR 2.1 (95\% CI: 1.1-3.9), $p=0.022$, for each additional point in the score]. Conclusions: Lung aeration as assessed by LUS on the 7th DOL may predict the development of sBPD.
\end{abstract}

\section{Introduction}

Bronchopulmonary dysplasia (BPD) is a major preterm morbidity and is strongly associated with poor neurodevelopmental outcomes [1]. Despite significant advances in neonatal care, the incidence of BPD has not decreased over the past decade $[2,3]$. Disrupted lung and pulmonary vascular development, infection and inflammation, postnatal

Supplementary information The online version of this article (https:// doi.org/10.1038/s41372-020-0724-z) contains supplementary material, which is available to authorized users.

Ignacio Oulego-Erroz

ignacio.oulego@gmail.com

1 Pediatric Intensive Care Unit, Complejo Asistencial Universitario de León, León, Spain

2 Working Group on Bedside Ultrasound of the Spanish Society of Pediatric Intensive Care (SECIP), Madrid, Spain

3 Biomedicine Institute of León (IBIOMED). University of León, León, Spain

4 Neonatal Intensive Care Unit, Complejo Asistencial Universitario de León, León, Spain insults (positive pressure ventilation, undernutrition, hyperoxia, etc.) to the developing lung together with a genetic background have been implicated in the pathophysiology of BPD [4-6]. With the exception of caffeine and vitamin A, most therapeutic interventions (nitric oxide, superoxide dismutase, permissive hypercapnia, etc.) tested in clinical trials have failed to demonstrate a positive effect in prevention of BPD despite robust pathogenic rationale for their use [7-9]. A large number of preterm infants with variable risk of developing BPD have been enrolled in these trials. Therefore, it is possible that potential beneficial effects of these therapies may have been diluted. A lesson learned from these negative trials is that patient selection based on disease phenotype and individual risk is important. In this line, there is a growing interest in finding early biomarkers of BPD that identify patients at risk in the initial phase of the pathological process and enable targeted therapies when they may be more effective. Some researchers have proposed that this "window of opportunity" encompasses the first 7-15 days of postnatal life, a concept that is supported by data from recent trials $[10,11]$. Therefore, ideally biomarkers should have the ability to identify high-risk patients within this time frame [12]. A biomarker has been defined as "any characteristic that is measured and evaluated as an 
indicator of normal biologic processes, pathogenic processes, or pharmacological responses to a therapeutic intervention" [13]. Biochemical, clinical, and radiological markers are currently being investigated, but most have either not shown sufficient accuracy or are not available in daily practice [14]. Lung ultrasound (LUS) is increasingly being used in the diagnosis and management of neonatal respiratory diseases [15]. Recently, Daniele De Luca's group has shown that a six-lung zone LUS score is highly accurate to predict the need for surfactant replacement in preterm infants below 30 weeks of gestational age (GA) with an area under the ROC curve (AUROC) of 0.94 [16]. Another study by Gregorio-Hernandez et al. using a slightly different score showed an AUROC of 0.97 for the prediction of CPAP failure and need for surfactant in preterm infants below 35 weeks. Importantly, in this study the AUROC of maximal $\mathrm{FiO}_{2}$ (a widely used parameter for the indication of surfactant) was 0.89 [17]. In this line, in a recent study by De Luca's group, surfactant replacement guided by the LUS score instead of $\mathrm{FiO}_{2}$ resulted in earlier therapy, less oxygen supplementation and less days of invasive ventilation compared with conventional management without increasing the number of surfactant doses [18].

As mentioned above, the pathogenesis of BPD is very complex. Lung pathology is variable and may include pulmonary vascular disease, tracheobronchomalacia, hyperinflation, patchy atelectasis, or cystic lesions, indicating that BPD is probably not a single disease [12]. However, a parenchymal component with loss of normal lung aeration is found in most patients with stablished BPD [19]. LUS has shown to be very sensitive in assessing lung aeration in different settings [20]. Surprisingly, studies assessing the role of LUS as a biomarker for developing BPD are very limited [21, 22]. According to data from the Neonatal Research Network, prediction of BPD in the first 3 days of life (DOL) is mostly determined by GA and from the 7th to the 28th DOL the influence of other factors reflecting postnatal lung injury such as ventilator support increases [23]. We hypothesize that loss of lung aeration early in postnatal life could be a biomarker of developing BPD. With this rationale, we chose to assess LUS score on the 7th DOL which we considered "early LUS" and investigated whether it can predict the development of moderate-severe BPD.

\section{Material and methods}

\section{Design and setting}

This was a prospective observational diagnostic accuracy study carried out in a third-level neonatal intensive care unit (NICU) at a university hospital.

\section{Patients}

Preterm infants born from the 1st of March 2017 to the 31st of October 2019 with a GA $<32$ weeks were considered eligible. Infants with major malformations, those who died before the 7th DOL and those who lacked parental informed consent were excluded. During the study period, respiratory care for preterm infants was provided according to the most recent update of the European Consensus Guidelines for respiratory support in very-low-birth-weight infants $[24,25]$. A systematic screening for hemodynamically significant patent ductus arteriosus (HsPDA) was performed in all patients between 48 and $96 \mathrm{~h}$ of life. The severity of ductal shunt was graded according to a multiparametric echocardiography score as none/mild, moderate, and severe. HsPDA was considered as a moderate-severe shunt and pharmacological closure was indicated per protocol in all cases of severe echocardiographic shunt or when shunt is moderate and there is hemodynamic and/or respiratory compromise attributable to PDA [26].

Data pertaining to the patients' baseline characteristics, duration, and level of respiratory support and associated morbidities were recorded prospectively until death or discharge.

\section{Lung ultrasound (LUS)}

An LUS was performed on the 7th DOL using a portable US machine (Sonosite M-Turbo, Fujifilm Japan) equipped with a linear-array probe (L25x, Sonosite, Fujifilm Japan). An experienced sonographer (PAQ) performed all LUS exams. This researcher has 5 years of clinical experience in neonatal LUS. The scanning protocol included the assessment of four lung zones in each lung (upper anterior, lower anterolateral, lower posterolateral, and lower posterior). Anterior zones were assessed in the supine positions, while for the assessment of the lower posterior lung zone, the patient was briefly positioned in lateral decubitus. A 5second clip was obtained in each lung zone and stored for offline analysis. LUS images were analyzed within $24 \mathrm{~h}$ by the investigator who performed the LUS exams. For each lung zone, lung aeration was assessed semiquantitatively from 0 to 3 points using the validated LUS score previously described by Danielle De Luca's group: [27] $0=$ normal lung with A line pattern and less than three B lines, $1=$ interstitial syndrome with more than three nonconfluent B lines, $2=$ severe interstitial syndrome with confluent B lines ("white lung" pattern) with or without small subpleural consolidations, and $3=$ extended consolidations (Fig. 1). The LUS aeration score was obtained by adding subscores in each lung zone (range 0-24 points). The LUS score was repeated on the 28th DOL to allow a performance comparison between "early" and "late" LUS. 


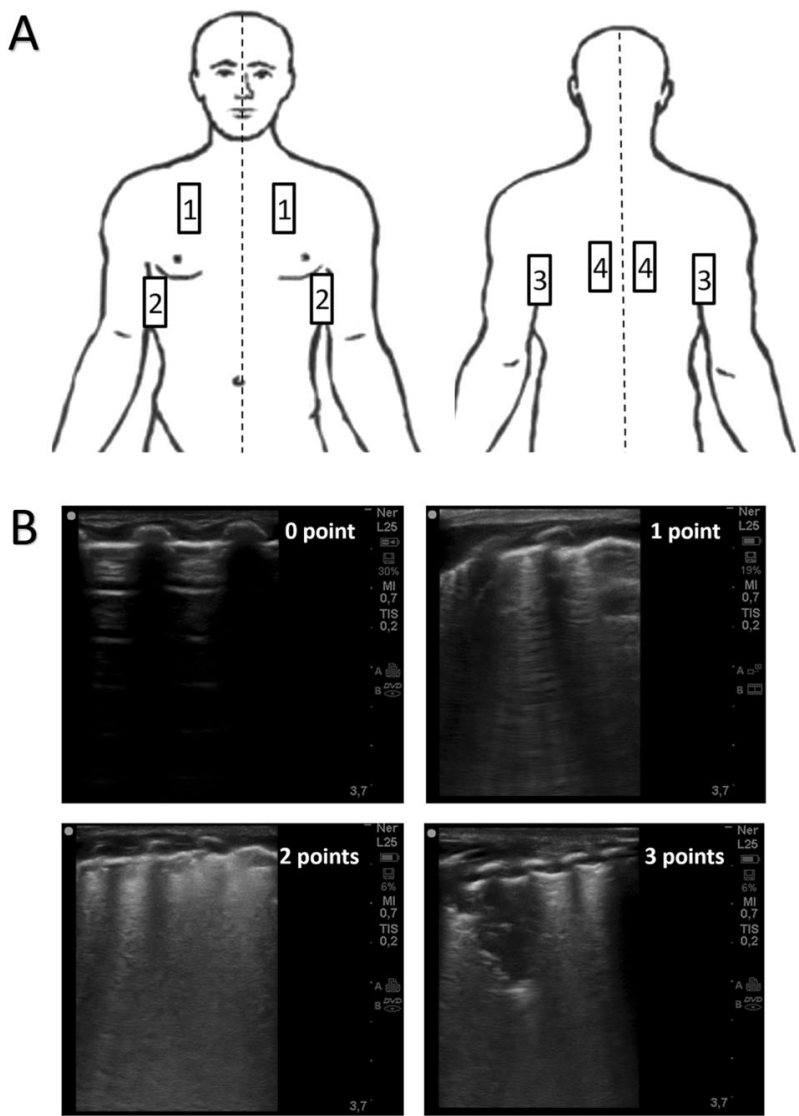

Fig. 1 Scanning protocol with aeration patterns and assigned punctuation for calculation of the LUS score. a Eight-zone scanning protocol. 1: anterosuperior (midclavicular line); 2 anterolateral (anterior axillary line); 3: posterolateral (posterior axillary line); 4: posterior (paravertebral). b Aeration pattern and assigned punctuations. 0 point (normally aerated lung with A line pattern), 1 point (mild interstitial syndrome with nonconfluent B-line pattern); 2 points (severe interstitial syndrome with confluent B-line pattern "white lung"); 3 point (extent lung consolidation).

To assess intra- and interobserver concordance [Cohen's $k$ coefficient and intra-class correlation coefficient (ICC)], the same investigator (PAQ) and a second blinded investigator (IOE) with 10-year experience in LUS reanalyzed the images in a random order and anonymously 7 days after LUS was originally obtained.

\section{Statistical analysis}

Categorical data are summarized as numbers (percentages), and quantitative data are summarized as medians (25-75th percentiles). Our primary endpoint was the diagnostic accuracy of LUS in predicting moderate-severe BPD (significant BPD, sBPD) according to the NIH workshop definition (mild BPD: at least 28 days of postnatal supplemental oxygen but free of oxygen or respiratory support at 36 weeks postmenstrual age (PMA); moderate BPD: need for oxygen with an inspired fraction $<30 \%$ at 36 weeks of postmenstrual age; severe BPD: need for an inspired fraction of oxygen $>30 \%$ or positive pressure ventilation at 36 weeks PMA) [28]. Accordingly, we divided the study population into two groups (non-sBPD and SBPD). The diagnosis of BPD was made by the responsible neonatologist who was blinded to the results of LUS. We also assessed the diagnostic accuracy of LUS for two additional endpoints: BPD of any grade (oxygen supplementation for more than 28 days) and CPAP dependency on the 28th DOL. Nonparametric tests were used to compare study groups as not all continuous variables were normally distributed.

We studied the relationship between the LUS score on the 7th DOL and the level of respiratory support provided including days of mechanical ventilation, respiratory index (RI), and duration of oxygen supplementation by using rhoSpearman correlation coefficients with and without adjustment for GA. The RI was calculated as the mean airway pressure multiplied by $\mathrm{FiO}_{2}$ and was used as a surrogate for ventilator support [10]. ROC curves were used to assess the diagnostic accuracy of the LUS score. Optimal cutoff points for sensitivity (Se) and specificity (Sp) were estimated from the ROC curve (point closest to 0,1 method) as well as positive $(\mathrm{LR}+)$ and negative $(\mathrm{LR}-)$ likelihood ratios. Logistic regression was used to assess whether the LUS score on the 7th DOL was independently associated with our primary outcome sBPD. Two models were fitted: one including sex, GA and LUS score, and a multivariate model including previously known risk factors for BPD which had an observed $p$ value of $<0.1$ in the univariate analysis.

According to an incidence of sBPD of $\sim 25 \%$ in our unit in recent years and assuming potential losses of $15 \%$ of the subjects, 12 subjects in the SBPD group and 27 subjects in the non-sBPD group would achieve a power of 0.9 with and type I error of 0.05 for a conservative target AUROC of 0.8 . A $p$ value $<0.05$ was considered statistically significant. SPSS v22 (IBM Corp., Armonk, NY, USA) statistical package was used for the analysis.

\section{Results}

Forty-two infants: $29(69.1 \%)$ in the non-sBPD group (no $\mathrm{BPD}=21$; mild $\mathrm{BPD}=8)$ and $13(30.9 \%)$ in the $\mathrm{sBPD}$ group (moderate $\mathrm{BPD}=9$; severe $\mathrm{BPD}=4$ ) were included. Sixteen patients were excluded for several reasons (Fig. 2). Excluded infants had similar baseline characteristics (birth weight, GA, and incidence of BPD) compared with included ones (data not shown). The patients' characteristics are shown in Table 1.

Infants in the sBPD group had higher LUS scores on the 7th [9 (8.5-12) vs. $4(3-5), p<0.001]$ and 28th DOL [8 $(7-12)$ vs. $3(1-4), p<0.001]$ than infants in the non-sBPD group. LUS scores on the 7 th and 28th DOL steadily 


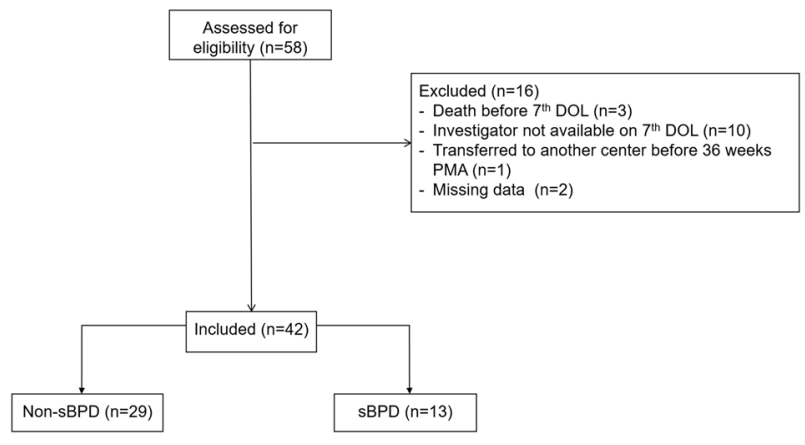

Fig. 2 Flow chart of the study. DOL day of life, PMA postmenstrual age.

increased accordingly to the grade of BDP at 36 weeks PMA ( $p$ for trend <0.001) (Fig. 3). The LUS score on the 7th DOL was significantly correlated with invasive mechanical ventilation days (rho $=0.534, p<0.001$ ), noninvasive mechanical ventilation days (rho $=0.594, p<$ 0.001 ), total days of mechanical ventilation (rho $=0.585$, $p<0.001$ ), maximal RI (rho $=0.616, p<0.001$ ), RI index on the 7th DOL (rho $=0.655, p<0.001$ ), and total days of oxygen supplementation (rho $=0.727, p<0.001)$. All results except for invasive mechanical ventilation days $(p=$ $0.154)$ remained significant $(p<0.01)$ after adjustment for GA. Children intubated on the 7th DOL had higher LUS score compared with children on noninvasive ventilation or oxygen therapy (Supplementary Fig. s1). The diagnostic accuracy of the LUS score for the primary and secondary outcomes is summarized in Table 2. The LUS score in the 7 th DOL had an area under the curve (AUC) of 0.94 (95\% CI 0.87-1) for the diagnosis of sBPD at 36 weeks of PMA. An optimal cutoff of $\geq 8$ points provided a Se, Sp, LR+, and LR - of 93\%, 91\%, 10.3 and 0.07, respectively (Supplementary Fig. s2). LUS score was also predictive of BPD of any grade [AUC 0.93 (CI 95\%: 0.86-1)] and CPAP dependency at 28th DOL [AUC $=0.89$ (95\% CI:0.74-1)]. The LUS on the 7th DOL score was independently associated with sBPD after adjustment for sex and GA [OR 1.7 (1.12-2.5), $p=0.011$; for each additional point in the score]. In the multivariate model, the LUS score was the only predictor of sBPD [OR 2.1 (1.1-3.9), $p=0.022$; for each additional point in the score] (Supplemental Material Table s1).

Intra-observer (Cohen's $k=0.92, \quad \mathrm{ICC}=0.997)$ and interobserver agreement (Cohen's $k=0.87, \mathrm{ICC}=0.995$ ) of the LUS interpretation were excellent.

\section{Discussion}

LUS is an inexpensive, noninvasive, and widely available tool for the evaluation of the neonatal lung [29]. In this
Table 1 Clinical characteristics of the study groups.

\begin{tabular}{|c|c|c|c|}
\hline Clinical characteristics & No $\operatorname{sBPD}(n=29)$ & $\operatorname{sBPD}(n=13)$ & $p$ \\
\hline Sex (male) & $19(65.5)$ & $10(76.9)$ & 0.460 \\
\hline Gestational age (weeks) & $29.5(28-31.5)$ & $27(25.7-28.2)$ & $<0.001$ \\
\hline Birth weight (g) & $1235(1110-1507)$ & $890(655-1130)$ & 0.000 \\
\hline Maternal chorioamnionitis & $2(6.9)$ & $4(30.7)$ & 0.041 \\
\hline Complete antenatal steroids & $20(68.9)$ & $9(69.2)$ & 0.986 \\
\hline Cesarean delivery & $18(62.1)$ & $8(61.5)$ & 0.974 \\
\hline Apgar $5^{\prime}$ & $9(8-10)$ & $8(5.5-9.5)$ & 0.090 \\
\hline CRIB $12 \mathrm{~h}$ & $1(0-2)$ & $2(1-7)$ & 0.002 \\
\hline Surfactant for RDS & $3(10.3)$ & $7(53.8)$ & 0.002 \\
\hline $\begin{array}{l}\text { Invasive mechanical } \\
\text { ventilation (days) }\end{array}$ & $0(0-7)$ & $7(0-23)$ & 0.000 \\
\hline $\begin{array}{l}\text { Invasive mechanical ventilation } \\
\text { (7th DOL) }\end{array}$ & $0(0)$ & $4(30.7)$ & 0.002 \\
\hline $\begin{array}{l}\text { Mechanical ventilation including } \\
\text { CPAP (days) }\end{array}$ & $5(2-10)$ & $37(12.5-57.5)$ & $<0.001$ \\
\hline RI (maximum) & $1.8(1.3-2.4)$ & $3.9(2.3-9.5)$ & 0.002 \\
\hline RI (7th DOL) & $0(0-1.2)$ & $2.1(1.6-2.7)$ & $<0.001$ \\
\hline HsPDA & $6(20.6)$ & $5(38.4)$ & 0.226 \\
\hline Late-onset sepsis & $4(13.8)$ & $6(46.1)$ & 0.023 \\
\hline Blood transfusion & $5(17.2)$ & $10(76.9)$ & $<0.001$ \\
\hline Number & $0(0-0)$ & $3(1-5)$ & 0.000 \\
\hline Inotropes-vasopressors & $5(17.2)$ & $10(76.9)$ & 0.000 \\
\hline VIS (maximum) & $7.5(3.7-10)$ & $5(5-11.2)$ & 0.859 \\
\hline $\begin{array}{l}\text { Oxygen supplementation } \\
\geq 28 \text { days (any grade BPD) }\end{array}$ & $8(33.3)$ & $13(100)$ & $<0.001$ \\
\hline Postnatal steroid use & $0(0)$ & $4(30.7)$ & $<0.001$ \\
\hline CPAP dependency 28th DOL & $1(3.4)$ & $8(61.5)$ & $<0.001$ \\
\hline IVH grade II-IV & $1(3.4)$ & $1(7.7)$ & 0.550 \\
\hline NEC & $0(0)$ & $3(23)$ & 0.007 \\
\hline Death before discharge & $0(0)$ & $0(0)$ & 1 \\
\hline
\end{tabular}

$\mathrm{RI}$ is calculated as mean airway pressure $\times \mathrm{FiO}_{2}$. VIS is calculated as dopamine + dobutamine $+(100 \times$ epinephrine $)+(100 \times$ norepinephrine $)+(10 \times$ milrinone $)$.

$C R I B$ clinical risk index for babies, $D O L$ day of life, IVH intraventricular hemorrhage, $N E C$ necrotizing enterocolitis, HsPDA hemodynamically significant patent ductus arteriosus in need for treatment, $R I$ respiratory index, $R D S$ respiratory distress syndrome, $V I S$ vasopressor-inotropic score (calculated only for those infants who received some inotrope or vasopressor).

prospective study, we showed that lung aeration assessed by a LUS score on the 7th DOL can accurately predict the development of moderate-severe BPD at 36 weeks of PMA.

The perinatal period until the 7-14th DOL is considered the window of opportunity for BPD prevention [30]. Interventions beyond this time frame, although important to preserve lung function, have a reduced chance of modifying the evolution of the disease in the short term. Therefore, research should focus on finding biomarkers that identify patients at risk of BPD early after birth. Previous research has been centered on biochemical biomarkers [31, 32]. The diagnostic accuracy of these markers has been only moderate, and most of them are still research tools that are not available in daily practice [32].

To our knowledge, only two previous studies have assessed a LUS score as an early biomarker for 

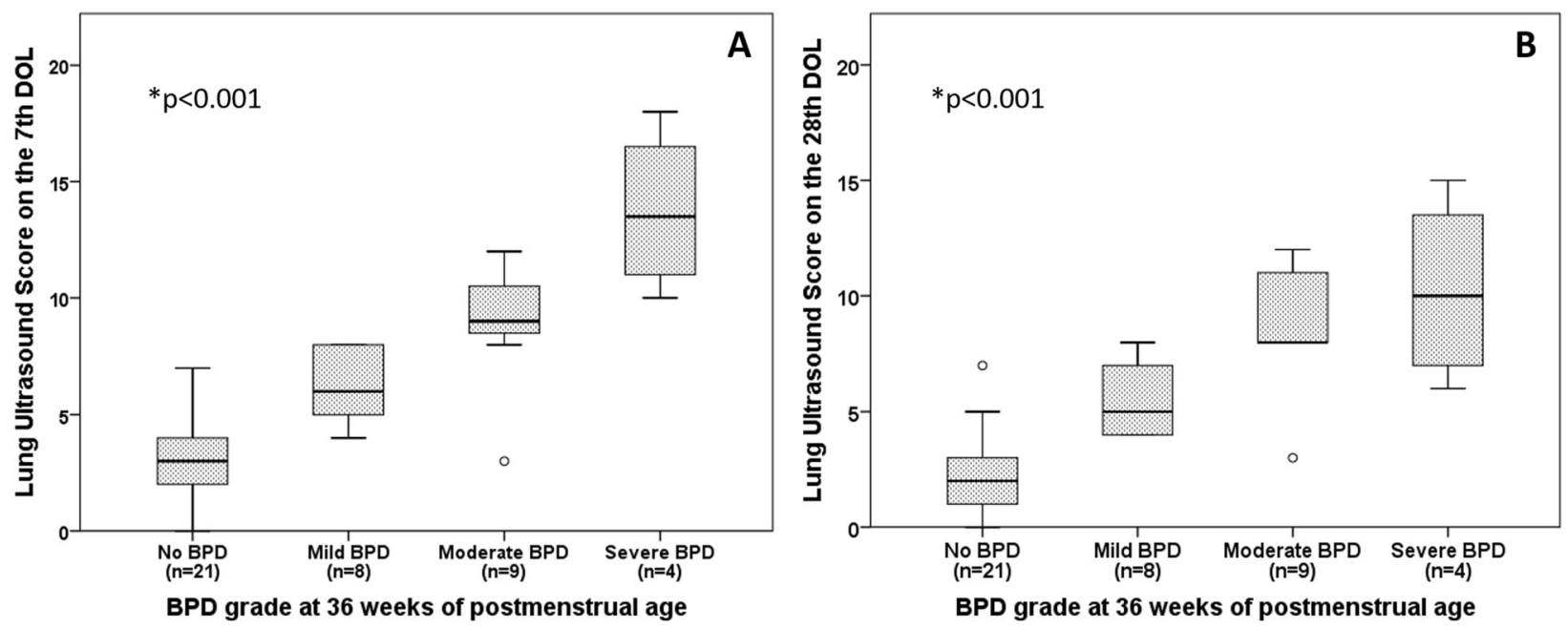

Fig. 3 Lung ultrasound score accorging to bronchopulmonary dysplasia grade at 36 weeks of postmenstrual age. Lung Ultrasound Score on the 7th (a) and 28th day of life (b) An asterisk indicates Jonckheere-Terpstra trend test.

Table 2 Diagnostic accuracy of the LUS score on the 7th and 28th DOL for the primary (moderate-severe BPD, sBPD) and secondary outcomes (BDP of any grade and CPAP dependency on the 28th DOL).

\begin{tabular}{lllrrrr}
\hline & AUC $(95 \%$ CI $)$ & Optimal cutoff & Se & Sp & LR+ & LR- \\
\hline LUS score (7th DOL) & & & & & & \\
sBPD & $0.94(0.87-1)$ & $\geq 8$ & 93 & 91 & 10.3 & 0.07 \\
Any grade BPD & $0.93(0.86-1)$ & $\geq 5$ & 90 & 81 & 3.6 & 0.12 \\
CPAP dependency & $0.89(0.74-1)$ & $\geq 9$ & 89 & 91 & 9.9 & 0.12 \\
LUS score (28th DOL) & & & & & & \\
sBPD & $0.92(0.83-1)$ & $\geq 6$ & 85 & 82 & 4.7 & 0.18 \\
Any grade BPD & $0.96(0.9-1)$ & $\geq 4$ & 95 & 86 & 6.8 & 0.06 \\
CPAP dependency & $0.97(0.91-1)$ & $\geq 8$ & 100 & 91 & 11.1 & 0 \\
\hline
\end{tabular}

Se sensitivity, $S p$ specificity, $L R+$ positive likelihood ratio, $L R-$ negative likelihood ratio. developing BPD. Alonso-Ojembarrena et al. performed LUS on the 1st DOL and 3rd DOL; postnatal weeks 1,2, 3 , and 4; and 36 weeks of PMA. They assessed six lung zones (upper anterior, lower-anterior, and lateral) and classified lung aeration using the same scoring system as the present study (range $0-18$ points). They found higher LUS scores from 1 week to 36 weeks PMA in infants who developed BPD of any grade. LUS on the 1 st $(\mathrm{AUC}=$ $0.8 ; \mathrm{Se} 70.6 \%, \mathrm{Sp} 79.2 \%)$ and 2 nd $(\mathrm{AUC}=0.93 ; \mathrm{Se}$ $73.7 \%$, Sp 100\%) week were moderately and highly predictive of any grade BPD respectively while LUS during the 4th week was moderately predictive (AUC = 0.89; Se 100\%, Sp 78.1\%) of moderate-severe BPD [21]. In this study, LUS was performed by an undetermined number of attending physicians, and posterior lung zones were not evaluated. In a retrospective study, Abdelmawala et al. performed eight-zone LUS at a median of 5 weeks of postnatal age (25th-75th percentile: 2-6 weeks) using the same scoring method as AlonsoOjembarrena and the present study. They found a higher LUS score in infants who developed moderate-severe
BPD and an AUC of 0.94 (Se 76\%, Sp 97\%). However, in the subset of infants who had their LUS performed during the first 2 weeks of life, the diagnostic accuracy of their chosen LUS score cutoff point was poor (Se 67\%, Sp $50 \%$ ) [22]. The interpretation of this study is difficult because LUS was not performed at a predefined time point and infants with HsPDA (a known risk factor for BPD) were excluded. Again, the authors did not assess posterior lung zones. Like other diffuse inflammatory lung diseases, such as bronchiolitis and "adult" acute RDS (ARDS), dependent lung zones are the most affected in BPD. Importantly, LUS scores have also shown promising results in predicting outcomes of these conditions [33-36]. The lack of assessment of posterior lung zones may explain the lower predictive value of early LUS in the aforementioned studies compared with our results. Alternatively, it is also possible that an early LUS score works better for the prediction of more severe grades of BPD. Based on our results, including the assessment of posterior lung zones, along with subgroup analysis according to BPD grade, may be considered in future 
studies to better delineate the role of LUS scores in predicting the development of BPD.

We considered that evaluation on the 7th DOL would be optimal from the perspective of BPD prevention. As shown by Alonso-Ojembarrena et al., performance of LUS too early after birth may not be discriminative, given that LUS findings in BPD are not specific and overlap with those of neonatal RDS [21]. By contrast, the identification of at-risk patients on the 7th DOL should be early enough to implement individualized therapy. For instance, early administration of corticosteroids (before or on the 7th DOL) has been shown to reduce BPD and mortality, while later use in ventilator-dependent infants does not [10, 37, 38]. However, universal application of early prophylactic postnatal steroids would expose a large number of preterm infants to complications and is not generally recommended. Individualized administration of postnatal steroids based on early LUS findings may be an attractive approach that is worth exploring.

Our study is limited by its single-center nature and small sample size. Therefore, our results should be considered preliminary. More importantly, to date the correlation between LUS findings and lung pathology in BPD has not been stablished. Similar LUS scoring systems have been successfully used in disparate lung diseases such as neonatal RDS, ARDS, pneumonia, or bronchiolitis [27, 33, 34, 39, 40]. Although this fact may confirm the validity of the technique in predicting outcomes, the diversity of diseases detected with this method leaves the clinician questioning how early LUS findings are tied to the pathogenesis of BPD. In our study late LUS had a worse predictive value for SBPD diagnosis compared with early LUS, suggesting that early LUS findings are not necessarily reflecting lung structural changes of stablished BPD but may rather indicate a previous phase of inflammatory acute lung injury characterized by increased water content and decreased aeration. However, LUS details not only the loss of aeration that may be caused by inflammation (ventilator induced injury, oxidative stress, etc.) in developing BPD but also that related to fluid overload, secondary infection, or recruitable atelectasis. How these superimposed conditions or their treatment may modify the LUS score and its predictive value has not been evaluated in our study. Another limitation is that we performed LUS at only two time points. Other time points should be explored as well as the combination of LUS with other biomarkers that may improve diagnostic accuracy. Finally, in our study, LUS was performed and analyzed by a unique highly experienced sonographer and, therefore, may lack external validity.

In conclusion, lung aeration as assessed by a LUS score on the 7th DOL may predict the development of moderate-severe BPD in preterm infants. The assessment of posterior lung zones seems to be important to improve diagnostic accuracy, but this hypothesis needs further exploration. More research is needed to evaluate the pathophysiological significance of early LUS changes in developing BPD. This is an essential step to assess if LUS can be used as a bedside biomarker to guide targeted therapies or design future trials.

Acknowledgements We wish to thank the nursing staff of our NICU and parents for their strength and patience

Author contributions IOE: conceived and designed the study, analyzed data, and drafted the paper. PAQ: conceived the study, acquired data, and critically reviewed the paper. STS: acquired data, analyzed data, and critically reviewed the paper. AJG: acquired data and critically reviewed the paper. SRB: acquired data and critically reviewed the paper. All authors gave their approval for the final version of the paper.

\section{Compliance with ethical standards}

Conflict of interest The authors declare that they have no conflict of interest.

Ethical approval The reference Institutional Review Board reviewed and approved the study protocol.

Informed consent Parents or legal guardians provided informed consent for participation.

Publisher's note Springer Nature remains neutral with regard to jurisdictional claims in published maps and institutional affiliations.

\section{References}

1. Cheong JLY, Doyle LW. An update on pulmonary and neurodevelopmental outcomes of bronchopulmonary dysplasia. Semin Perinatol. 2018;42:478-84.

2. Lui K, Lee SK, Kusuda S, Adams M, Vento M, Reichman B, et al. Trends in outcomes for neonates born very preterm and very low birth weight in 11 high-income countries. J Pediatr. 2019;215:32-40.

3. Webbe JWH, Duffy JMN, Afonso E, Al-Muzaffar I, Brunton G, Greenough A, et al. Core outcomes in neonatology: development of a core outcome set for neonatal research. Arch Dis Child Fetal Neonatal Ed. 2019. https://doi.org/10.1136/archdischild-2019317501.

4. Bancalari E, Claure N, Sosenko IRS. Bronchopulmonary dysplasia: changes in pathogenesis, epidemiology and definition. Semin Neonatol SN. 2003;8:63-71.

5. McEvoy CT, Jain L, Schmidt B, Abman S, Bancalari E, Aschner JL. Bronchopulmonary dysplasia: NHLBI Workshop on the Primary Prevention of Chronic Lung Diseases. Ann Am Thorac Soc. 2014;11 Suppl 3:S146-S153.

6. McEvoy CT, Aschner JL. The natural history of bronchopulmonary dysplasia: the case for primary prevention. Clin Perinatol. 2015;42:911-31.

7. Askie LM, Ballard RA, Cutter GR, Dani C, Elbourne D, Field D, et al. Inhaled nitric oxide in preterm infants: an individual-patient data meta-analysis of randomized trials. Pediatrics. 2011; 128:729-39. 
8. Davis JM, Parad RB, Michele T, Allred E, Price A, Rosenfeld W, et al. Pulmonary outcome at 1 year corrected age in premature infants treated at birth with recombinant human $\mathrm{CuZn}$ superoxide dismutase. Pediatrics. 2003;111:469-76.

9. Dunn MS, Kaempf J, de Klerk A, de Klerk R, Reilly M, Howard $\mathrm{D}$, et al. Randomized trial comparing 3 approaches to the initial respiratory management of preterm neonates. Pediatrics. 2011;128:e1069-e1076.

10. Onland W, Cools F, Kroon A, Rademaker K, Merkus MP, Dijk $\mathrm{PH}$, et al. Effect of hydrocortisone therapy initiated 7 to 14 days after birth on mortality or bronchopulmonary dysplasia among very preterm infants receiving mechanical ventilation: a randomized clinical trial. JAMA. 2019;321:354-63.

11. Doyle LW, Cheong JL, Ehrenkranz RA, Halliday HL. Early (<8 days) systemic postnatal corticosteroids for prevention of bronchopulmonary dysplasia in preterm infants. Cochrane Database Syst Rev. 2017;10:CD001146.

12. Lal CV, Ambalavanan N. Biomarkers, early diagnosis, and clinical predictors of bronchopulmonary dysplasia. Clin Perinatol. 2015;42:739-54.

13. Biomarkers Definitions Working Group. Biomarkers and surrogate endpoints: preferred definitions and conceptual framework. Clin Pharmacol Ther. 2001;69:89-95.

14. Alvarez-Fuente M, Moreno L, Lopez-Ortego P, Arruza L, AvilaAlvarez A, Muro M, et al. Exploring clinical, echocardiographic and molecular biomarkers to predict bronchopulmonary dysplasia. PloS One. 2019;14:e213210.

15. Hiles M, Culpan A-M, Watts C, Munyombwe T, Wolstenhulme S. Neonatal respiratory distress syndrome: chest X-ray or lung ultrasound? A Syst Rev Ultrasound Leeds Engl. 2017;25:80-91.

16. De Martino L, Yousef N, Ben-Ammar R, Raimondi F, ShankarAguilera S, De Luca D. Lung ultrasound score predicts surfactant need in extremely preterm neonates. Pediatrics. 2018;142. https:// doi.org/10.1542/peds.2018-0463.

17. Gregorio-Hernández R, Arriaga-Redondo $\mathrm{M}$, Pérez-Pérez A, Ramos-Navarro C, Sánchez-Luna M. Lung ultrasound in preterm infants with respiratory distress: experience in a neonatal intensive care unit. Eur J Pediatr. 2020;179:81-89.

18. Raschetti R, Yousef N, Vigo G, Marseglia G, Centorrino R, BenAmmar R, et al. Echography-guided surfactant therapy to improve timeliness of surfactant replacement: a quality improvement project. J Pediatr. 2019;212:137-143. e1

19. Walkup LL, Woods JC. Newer imaging techniques for bronchopulmonary dysplasia. Clin Perinatol. 2015;42:871-87.

20. Mojoli F, Bouhemad B, Mongodi S, Lichtenstein D. Lung ultrasound for critically Ill patients. Am J Respir Crit Care Med. 2019;199:701-14.

21. Alonso-Ojembarrena A, Lubián-López SP. Lung ultrasound score as early predictor of bronchopulmonary dysplasia in very low birth weight infants. Pediatr Pulmonol. 2019;54:1404-9.

22. Abdelmawla M, Louis D, Narvey M, Elsayed Y. A lung ultrasound severity score predicts chronic lung disease in preterm infants. Am J Perinatol. 2019;36:1357-61.

23. Laughon MM, Langer JC, Bose CL, Smith PB, Ambalavanan N, Kennedy KA, et al. Prediction of bronchopulmonary dysplasia by postnatal age in extremely premature infants. Am J Respir Crit Care Med. 2011;183:1715-22.

24. Sweet DG, Carnielli V, Greisen G, Hallman M, Ozek E, Plavka R, et al. European consensus guidelines on the management of respiratory distress syndrome-2016 update. Neonatology. 2017; 111:107-25.
25. Sweet DG, Carnielli V, Greisen G, Hallman M, Ozek E, Te Pas A, et al. European consensus guidelines on the management of respiratory distress syndrome-2019 update. Neonatology. 2019;115:432-50.

26. Rodríguez-Blanco S, Oulego-Erroz I, Alonso-Quintela P, TerrobaSeara S, Jiménez-González A, Palau-Benavides M. N-terminalprobrain natriuretic peptide as a biomarker of moderate to severe bronchopulmonary dysplasia in preterm infants: a prospective observational study. Pediatr Pulmonol. 2018;53:1073-81.

27. Brat R, Yousef N, Klifa R, Reynaud S, Shankar Aguilera S, De Luca D. Lung ultrasonography score to evaluate oxygenation and surfactant need in neonates treated with continuous positive airway pressure. JAMA Pediatr. 2015;169:e151797.

28. Jobe AH, Bancalari E. Bronchopulmonary dysplasia. Am J Respir Crit Care Med. 2001;163:1723-9.

29. Sharma D, Farahbakhsh N. Role of chest ultrasound in neonatal lung disease: a review of current evidences. J Matern-Fetal Neonatal Med J Eur Assoc Perinat Med Fed Asia Ocean Perinat Soc Int Soc Perinat Obstet. 2019;32:310-6.

30. Álvarez-Fuente M, Moreno L, Mitchell JA, Reiss IK, Lopez P, Elorza D, et al. Preventing bronchopulmonary dysplasia: new tools for an old challenge. Pediatr Res. 2019;85:432-41.

31. Förster K, Sass S, Ehrhardt H, Mous DS, Rottier RJ, Oak P, et al. Early identification of bronchopulmonary dysplasia using novel biomarkers by proteomic screening. Am J Respir Crit Care Med. 2018;197:1076-80.

32. Rivera L, Siddaiah R, Oji-Mmuo C, Silveyra GR, Silveyra P. Biomarkers for bronchopulmonary dysplasia in the preterm infant. Front Pediatr. 2016;4:33.

33. Bueno-Campaña M, Sainz T, Alba M, Del Rosal T, MendezEchevarría A, Echevarria R, et al. Lung ultrasound for prediction of respiratory support in infants with acute bronchiolitis: a cohort study. Pediatr Pulmonol. 2019;54:873-80.

34. Zoido Garrote E, García Aparicio C, Camila Torrez Villarroel C, Pedro Vega García A, Muñiz Fontán M, Oulego Erroz I. Usefulness of early lung ultrasound in acute mild-moderate acute bronchiolitis. a pilot study. An Pediatr. 2019;90:10-18.

35. Bello G, Blanco P. Lung ultrasonography for assessing lung aeration in acute respiratory distress syndrome: a narrative review. J Ultrasound Med J Am Inst Ultrasound Med. 2019;38:27-37.

36. Chiumello D, Umbrello M, Sferrazza Papa GF, Angileri A, Gurgitano M, Formenti P, et al. Global and regional diagnostic accuracy of lung ultrasound compared to $\mathrm{CT}$ in patients with acute respiratory distress syndrome. Crit Care Med. 2019;47:1599-606.

37. Doyle LW, Cheong JL, Ehrenkranz RA, Halliday HL. Early (< 8 days) systemicpostnatal corticosteroids for prevention of bronchopulmonary dysplasia inpreterm infants. Cochrane Database Syst Rev. 2017:24;10.

38. Morris IP, Goel N, Chakraborty M. Efficacy and safety of systemic hydrocortisone for the prevention of bronchopulmonary dysplasia in preterm infants: a systematic review and metaanalysis. Eur J Pediatr. 2019;178:1171-84.

39. Bouhemad B, Brisson H, Le-Guen M, Arbelot C, Lu Q, Rouby JJ. Bedside ultrasound assessment of positive end-expiratory pressure-induced lung recruitment. Am J Respir Crit Care Med. 2011;183:341-7.

40. Li LM, Li LH, Guan J, Yang Q, Han JQ, Chao YG. The value of lung ultrasound score for therapeutic effect assessment of ventilator-associated pneumonia. Zhonghua Nei Ke Za Zhi. 2016;55:950-2. 